\title{
Oscillator with Hyperbolically Variable Inertia
}

\author{
Zvonko RAKARIĆ, Boris STOJIĆ*
}

\begin{abstract}
Novel oscillator model is introduced whose kinetic energy is inversely proportional to the generalized coordinate. System is referred to as "Oscillator with Hyperbolically Variable Inertia" (OHVI), since its inertia undergoes hyperbolic growth. Concept of comparison of free OHVI with the behaviour of simple harmonic oscillator (HO) via contraction and deformation of a 3D energy surface is introduced. Analysis of phase orbits and fixed point is performed. Obtaining an exact analytical solution for motion of free $\mathrm{OHVI}$ is shown. Main features of the system are demonstrated by numerical experiment performed for kinematically excited OHVI using appropriate mechanism design. It is shown that OHVI has an exact solution in the form of Jacoby elliptic function. Also, phase orbits of OHVI show characteristic oval ("egglike") shape. Motiontime histories and amplitude-frequency (AF) characteristics for forced oscillations were determined. Analysis of solutions for free system indicates possibility of realization of oscillatory system that has very long period i.e. low natural frequency. Comparison of system behaviour with typical nonlinear oscillator with restoring force of hardening type was carried out. Presented analysis shows that such system can be successfully applied for attenuation of low frequency vibrations or their detection
\end{abstract}

Keywords: energy surface contraction; harmonic excitation of support; nonlinear oscillator; singular inertial coefficient; super-attenuation

\section{INTRODUCTION}

Thus far, a lot of work has been done concerning "pseudo oscillator", for which restoring force is inversely proportional to the generalized coordinate (see, for instance, $[1,2])$. In these studies, inertial term is the same as in the simple harmonic oscillator (HO). Motivated by these investigations, in the present work reverse concept is considered, i.e. the system is introduced that has the same form of restoring force as in the $\mathrm{HO}$, but generalized inertial coefficient inversely proportional to the generalized coordinate. Simulation of motion of such system shows oscillatory behaviour with possibility of achieving low and ultra-low natural frequency. Several different mechanisms that are known for such features were discussed in previously published papers. Some of them are folded pendulum mechanism [3] and low frequency pendulum mechanism [4]. For the first one, developed in 1962 [5], many related papers have been published so far as well as many different technical applications (see [6] and paper cited in it). Second type of mechanism (low frequency pendulum) was developed later (in [4]) but its technical realization is still being waited for. It should be emphasized that, in contrast to thoroughly studied vibrations that occur in the frequency range of about $1 \mathrm{~Hz}$ or higher, fields of vibrations well below $1 \mathrm{~Hz}$ are still insufficiently investigated [7]. There are many reasons why it is of interest to cover this vibration field by both good theoretical understanding and practical devices. Some of important practical applications are e.g. structural health monitoring (SHM) [8], vibration isolation [9], vibration detection [10] and energy harvesting of ambient energy [11]. Decreasing the natural frequency of structures is, as it is well known, a common way in vibration isolation problems. In case of linear systems this can be achieved by reducing stiffness, but at the cost of too large static deflection, which imposes significant limitations to this approach [12]. For this reason, intensive work is being carried out to find solutions by employing system nonlinearity [13]. Proposing utilization of variable inertia of the basic system, this paper aims to give further contribution in the field of non-linearity exploitation for vibration isolation. The emphasis here is on passive changing of inertia (i.e. system inertia changes as a consequence of its motion). Systems with such property can be treated as a Passive Variable Inertia Vibratory Systems (PVIVS), and could present a new way of thinking in this field. Although here the demonstration of principles is shown by using real mechanical structure example, authors believe that this concept should be extended to other non-mechanical systems and applied universally. To this end, it is also the way this approach is presented here. At this point it is also worth noticing that, from mechanical standpoint, nonlinearities can in general be of geometric or material origin, or their combination [12]. Although investigation demonstrated herein relies exclusively on employing geometric nonlinearities, using material nonlinearities to achieve described effect may be of great importance too. Hence, potentials of introduced approach in the field of modern metamaterials and metastructures in vibration and sound isolation are worth further investigations.

The rest of this paper is organized as follows. In Section 2, the mathematical model of the system is introduced and discussed. The governing equation of free conservative oscillator is presented. Comparison with simple harmonic oscillator (HO) is given in Section 3 by introducing concept of contraction and deformation of $3 \mathrm{D}$ energy surface of HO. Deriving exact solution of motion for free conservative system is presented in Section 4, where expression for natural frequency is also given. Discussion about solution of motion is also provided in this section. Demonstration of introduced approach by using specific design of mechanical system subjected to external periodic excitation is given in Section 5. Finally, in Section 6 conclusions and possible directions of further research are given.

\section{MATHEMATICAL MODEL OF THE SYSTEM}

A conservative system with a single degree of freedom (SDOF) is considered whose motion with respect to the stable equilibrium position is described by a generalized coordinate $q$.

Introduced concept assumes that the kinetic energy has the following form: 
$E_{k}=\frac{1}{2} I_{0}(q) \dot{q}^{2}=\frac{1}{2} \frac{\hbar}{q} \dot{q}^{2}$

Overdot stands for derivation with respect to time $t$. $I_{0}(q)$ is generalized inertial coefficient that depends on generalized coordinate $q$. It is assumed that the parameter $\hbar$ is constant and depends on parameters of the system. Clearly, dimension of parameter $\hbar$ depends on the nature of the generalized coordinate. It should be emphasized here that, to the authors' knowledge, the form of kinetic energy defined by Eq. (1) is novel i.e. does not appear in previous publications.

Further, according to introductory assumptions, the potential energy has the same form as in the simple HO:

$E_{p}=\frac{1}{2} k_{e k v} q^{2}$

where $k_{e k v}$ stands for equivalent stiffness coefficient of the system.

It can be seen from Eq. (1) that the only term that exists in the denominator of $I_{0}(q)$ is generalized coordinate $q$ in linear form, as assumed by introducing the regarded concept. Because of that, inertial term obeys hyperbolic growth for which reason the regarded system will be referred to as "oscillator-with-hyperbolically-variableinertia" (OHVI).

Based on Eq. (1) and Eq. (2), the equation of motion reads:

$\frac{\hbar}{q} \ddot{q}-\frac{1}{2} \frac{\hbar}{q^{2}} \dot{q}^{2}+k_{e k v} q=0$

The system described by Eq. (3) is essentially nonlinear. Namely, inertial coefficient in kinetic energy term cannot be expanded into series and linearized. In fact, such inertial coefficient as $I_{0}(q)$ given by Eq. (1) can present, for example, the first term of singular or principal part of the Laurent series of a much more complex inertial coefficient. Anyway, there is a singularity here due to the hyperbolic growth of $I_{0}(q)$, in a way that it tends to infinity for a finite value of generalized coordinate, $I_{0}(q) \rightarrow \infty$ for $q \rightarrow 0$. Unlike linear systems with singular coefficients, which can be analyzed by using some of numerous and well developed available methods [14], system under investigation is highly nonlinear, so consequently appropriate technique for further analysis has to be figured out. The idea applied herein is based on the following thoughts: comparing the form of potential and kinetic energy of the system governed by Eq. (3) with those of the $\mathrm{HO}$, the former can be thought of as a certain modification of the latter. Therefore it can be useful to conduct qualitative analysis that will result in determination of relationship between $\mathrm{HO}$ and the system regarded. This analysis is given in Section 3.

\section{FROM HO TO OHVI VIA CONTRACTION OF ENERGY SURFACE}

Kinetic energy given by Eq. (1) imposes certain conditions that have to be satisfied. These conditions are:
Condition 1: In order to provide finite value of kinetic energy at $q=0$, velocity in equilibrium position has to be zero:

$\dot{q}(q=0) \equiv 0$

Keeping in mind that generalized coordinate $q$ is measured from the stable equilibrium, condition given by Eq. (4) implies that the equilibrium position is at the same time the stop position.

Condition 2: Taking into account that the coefficient $I_{0}(q)$ has to be positive (kinetic energy is a positive definite function), observing form of $I_{0}(q)$ defined by Eq. (1) it follows:

$$
I_{0}(q)>0 \rightarrow q>0
$$

Condition given by Eq. (5) means that motion takes place only on one side of the equilibrium position.

Taking into account conditions imposed on the system, as well as that total energy of the conservative system is given by the expression:

$E=\frac{1}{2} \frac{\hbar}{q} \dot{q}^{2}+\frac{1}{2} k_{e k v} q^{2}=\mathrm{const}$

the diagram of energy surface for OHVI is plotted in Fig. 1a together with the diagram of energy for $\mathrm{HO}$, as a function of $q$ and $\dot{q}$. The shape of energy surface of HO is clear and well known. Energy surface of OHVI with respect to that of the $\mathrm{HO}$ is contracted to the positive region of $q$ and deformed. This deformation is also manifested by distortion of phase orbits (Fig. 1b); elliptic orbits in the case of HO become oval (egglike) for OHVI.

Phase orbits of OHVI (red thick line in Fig. 1b) show shortening with respect to the HO. Figure shows that, with respect to the $\mathrm{HO}$, range of values of generalized coordinate is limited and, at the same time, values of velocity are decreased too. Symmetry exists only to the $E$ $-q$ plane. For the same initial conditions (point A in Fig. $1 \mathrm{~b}, \mathrm{OHVI}$ and HO have the same unchanged amount of energy. However, on the basis of phase orbits it is clear that two systems have completely different ways of motion. So, Fig. 1 illustrates the essence of introduced form of kinetic energy defined by Eq. (1) and its influence on the system behaviour.

The point $\mathrm{B}$ is a fixed point. From a purely mathematical standpoint, during the motion in the phase plane, point that represents the instantaneous state of the system will asymptotically tend to fixed point and will never reach it. Taking this into account, and without violating the theorem of uniqueness of solution to the ordinary differential equations, it can be concluded that the solution on the part $\mathrm{A}-\mathrm{B}^{\prime}$ does exist, where $\mathrm{B}^{\prime}$ is a point in arbitrary small surroundings of fixed point B. From a practical point of view for such free conservative system, it is sufficient that solution existence on the part A- B' is deduced. Additionally, with external influence on this system, it will be possible for it to settle and subsequently pass through the fixed point of the basic system. Rigorous 
mathematical treatment of those considerations exceeds the scope of this paper and will therefore be omitted.
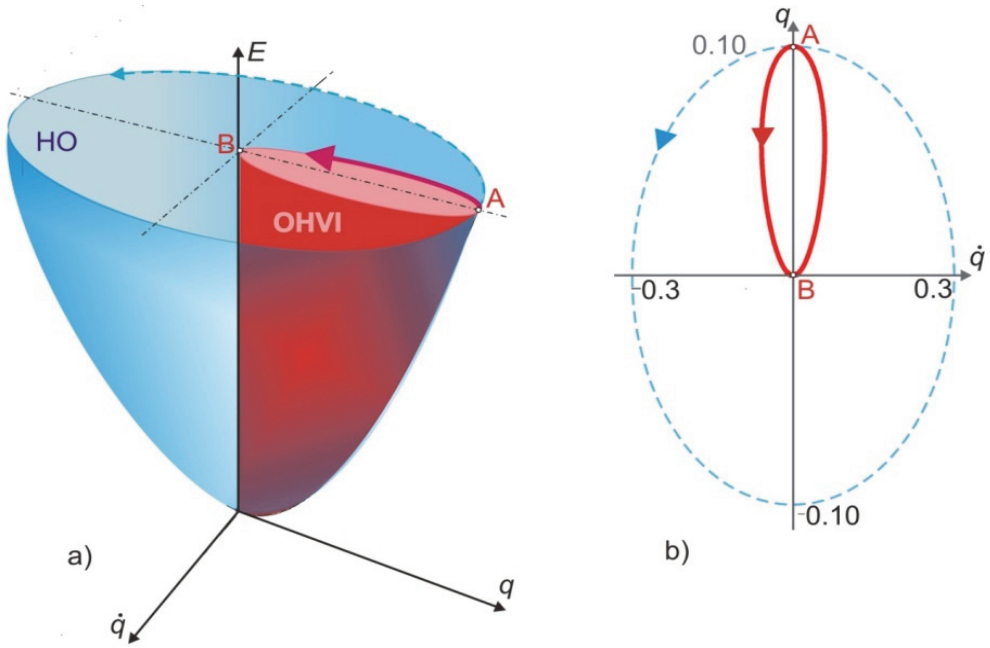

b)

Figure 1a) 3D diagrams of energy surface $E(q, \dot{q})$ for: $\mathrm{HO}$ (transparent, larger external surface); OHVI (red, smaller, internal surface); b) Phase orbits $(q(0)=0.1$ and $\dot{q}(0)=0$ ) for: HO (blue, dashed line); OHVI (red thick solid line)

\section{EXACT SOLUTION OF MOTION}

In order to obtain the solution of equation of motion (Eq. (3)), a new generalized coordinate $Q$ is introduced:

$Q=\int \sqrt{\frac{1}{2} \frac{\hbar}{q} d q}$

After performing integration in Eq. (7), a relation is derived between coordinates $Q$ and $q$ :

$Q^{2}=2 \hbar q$

It is assumed that $q(0)=Q(0)=0$. From Eq. (8) follows that $q(t)$ has to be greater than zero, which is in compliance with condition 2 given by Eq. (5). On the other hand, such assumption on $Q$ does not exist. In this place it is interesting to note that, in mathematical sense, Eq. (8) presents a suitable replacement of variables in order to solve an ODE, but in physical sense it may reflect the link between some two coordinates in the system that is considered. Namely, as it is known, any coordinate may be chosen as a generalized coordinate, but suitability of derived solution of motion for different choices may vary depending on what is chosen as the main analysis focus [15].

Further, from Eq. (7) follows:

$$
\frac{\mathrm{d} Q}{\mathrm{~d} t}=\sqrt{\frac{1}{2} \frac{\hbar}{q} \frac{\mathrm{d} q}{\mathrm{~d} t}}
$$

$\frac{\mathrm{d}^{2} Q}{\mathrm{~d} t^{2}}=\sqrt{\frac{1}{2} \frac{\hbar}{q} \frac{\mathrm{d}^{2} q}{\mathrm{~d} t^{2}}}-\frac{1}{2} \sqrt{\frac{\hbar}{2} q^{-3 / 2}\left(\frac{\mathrm{d} q}{\mathrm{~d} t}\right)^{2}}$

Substituting Eq. (9a), Eq. (9b) in Eq. (3), after rearranging, the following "new" nonlinear and nonsingular equation is derived:

$$
\frac{\mathrm{d}^{2} Q}{\mathrm{~d} t^{2}}+\frac{k_{e k v}}{4 \hbar^{2}} Q^{3}=0
$$

System governed by Eq. (10) is known as a pure cubic oscillator. This equation has an exact solution in the form of Jacobi elliptic function [16]. Using this fact, as well as Eq. (8), the exact solution to Eq. (3) reads:

$$
q(t)=A_{q} \mathrm{cn}^{2}\left[\sqrt{\frac{A_{q} k_{e k v}}{2 \hbar} t}-\phi_{q}, \frac{1}{2}\right]
$$

$A_{q}$ and $\phi_{q}$ in Eq. (11) are amplitude and phase respectively, and they are determined by initial conditions, $q(0)=q_{0}$ and $\dot{q}(0)=\dot{q}_{0}$.

The solution given by Eq. (11) is continuous in time, i.e. it exists for every $t$. As it was mentioned in the Section 3 , here it is sufficient to be continuously on path (AB). Motion between these positions can with certainty be achieved physically.

On the basis of Jacoby elliptic function property, period of general system described by Eq. (3) is given by [16]:

$T_{q}=2 K(1 / 2) \sqrt{\frac{2 \hbar}{k_{e k v}} A_{q}^{-1 / 2}}$

In Eq. (12), $K(1 / 2)$ is complete elliptic integral of the first kind.

In Fig. 2, diagrams of motions and velocities are shown in case of HO and OHVI. Significant period increase (i.e. frequency decrease) in case of OHVI in comparison to HO is apparent, despite of coordinate's range shortening. This follows from low velocity due to the contraction of energy surface, as phase orbits show (Fig. 1b).

Illustration of system behaviour given by Fig. 2 shows that, when compared to the HO of comparable size, OHVI is capable of achieving both low frequency and low velocity. Since stop position coincides with equilibrium 
position, analogy with overdamped oscillator can be established although in this case there is no damping. Such behaviour can be viewed as a super-attenuation feature, which is also noted in Fig. 2.

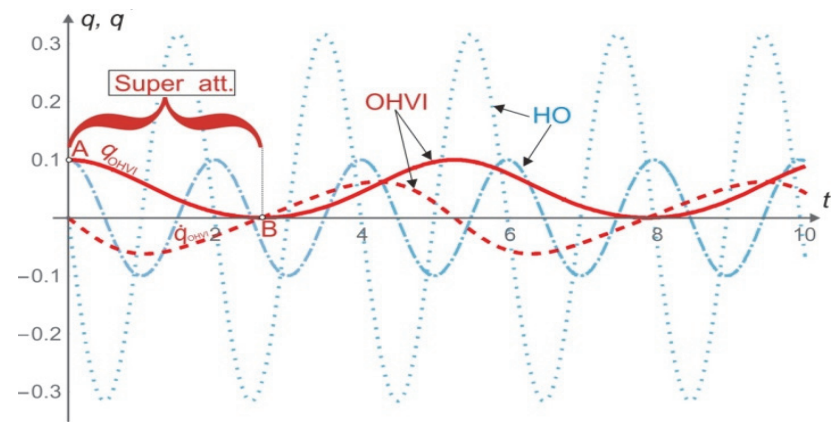

Figure 2 Diagrams for motion for $\mathrm{HO}$ (blue Dot-dashed line) and OHVI (solid thick red line). Also, time history of velocities are given (HO-blue dotted line; OHVI-red dashed line). Parameters: $\hbar=2, k_{e k v}=20, q(0)=0.1, \dot{q}(0)=0$ Observe super-attenuation feature of $\mathrm{OHVI}$.

\section{FORCED OSCILLATIONS OF MECHANICAL PLATFORM AS OHVI SYSTEM}

As a possible real-world example of a system with OHVI feature, mechanical system shown in Fig. 3 is considered. Heavy platform $\mathrm{AB}$, whose mass is $m$, is connected to the support by massless rigid rod and massless slider $D$. Platform is further connected to another massless rigid rod OC by hinge in point C. Rod OC itself is also connected to the platform by hinge in point $\mathrm{O}$. Platform can hence move independently with respect to the support in a way that relative motion has one degree of freedom. (As an application example, it can e.g. be

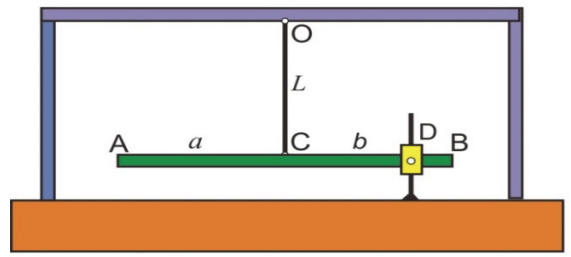

a) supposed that platform carries some sensitive equipment that needs to be isolated from the environment in dynamical sense.)

Analysis will be carried out with assumption that the support moves periodically in horizontal direction with the amplitude $X_{0}$, according to the harmonic law:

$$
x(t)=X_{0} \sin \Omega t
$$

As a generalized coordinate $q$, an angle of the rod OC with respect to the vertical axis is chosen. For deriving equation of motion for the platform, Lagrange equation of the second kind will be used. For that purpose, in the first step kinetic energy is determined. On the basis of Fig. 3, it applies:

$$
\begin{aligned}
& x_{c}=x+L \sin q \\
& y_{c}=-L \cos q \\
& b \cos \alpha=b-L \sin q
\end{aligned}
$$

Kinetic energy of the platform is:

$$
\begin{aligned}
& E_{k}=\frac{1}{2} m\left(\dot{x}^{2}+2 L \dot{x} \dot{q} \cos q\right)+ \\
& +\frac{1}{2}\left(m L^{2}+\frac{J_{c} L \cos ^{2} q}{2 b \sin q-L \sin ^{2} q}\right) \dot{q}^{2}=E_{k}^{\text {forced }}+E_{k}^{\text {free }}
\end{aligned}
$$

With $J_{c}$, mass moment of inertia of the platform for its center of mass is denoted.

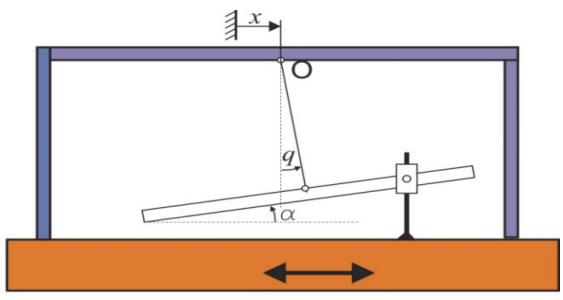

b)

Figure 3 Example of system with OHVI feature; a) Free system; b) Forced oscillations due to harmonic excitation of support given by $x(t)$ (Eq. (13)).

Eq. (17) shows that kinetic energy contains two parts. The first one is due to the motion of the point $\mathrm{O}$, i.e., due to the motion of the support given by Eq. (13). This part is denoted by $E_{k}^{\text {forced }}$. When the support is at rest, then $\dot{x}=0$, and only second term in Eq. (17) exists and this term corresponds to the free motion of the platform. Expanding the second term of Eq. (17) around $q=0$ into Laurent series, which is appropriate to be used instead of Taylor series because of system singularity, reads:

$E_{k}^{\text {free }}=\left[\frac{1}{2}\left(\frac{J_{c} L}{2 b}\right) \frac{1}{q}+(\ldots) q^{0}+(\ldots) q+\ldots\right] \cdot \dot{q}^{2}$

Keeping only the first term inside the brackets in Eq. (18), it can be observed that it corresponds to the starting form of kinetic energy given by Eq. (1), i.e. it has the same form as the coefficient before generalized coordinate squared. Retaining only the first term in Eq. (18) is justified in the case of small values of generalized coordinate. In this case, it is assumed that the platform performs small oscillations with respect to the support.

By applying $\cos q \approx 1-\frac{q^{2}}{2}+\ldots$, potential energy of the system under current assumptions becomes

$$
E_{p}=-m g L \cos q \approx-m g L\left(1-\frac{q^{2}}{2}\right)=-m g L+\frac{1}{2} m g L q^{2}
$$

Comparing Eq. (19) to Eq. (2), it can be noticed that expressions for the potential energy given by these equations have the same form up to the constant term $m g L$, which anyway disappears while deriving Lagrange's equation of motion of second kind.

For derivation of final form of equation of motion, Eq. (18) is introduced to Eq. (17) so that only first term inside 
the brackets is taken into account. By using these equations as well as Eq. (19) and expression (13) that define exciting motion, the equation of motion of the platform is derived:

$$
\frac{J_{c}}{2 b} \frac{1}{q} \ddot{q}-\frac{1}{2} \frac{J_{c}}{2 b} \frac{1}{q^{2}} \dot{q}^{2}+m g q=m X_{0} \Omega^{2} \sin \Omega t
$$

Eq. (20), as Eq. (3), is highly nonlinear and involves singularity, but now it is also nonhomogeneous, due to the external kinematic excitation. Due to this nonhomogeneity, introduction of the new variable such as that introduced by Eq. (8) will not lead to a suitable form, as was the case with Eq. (10) that was convenient for analytical consideration. Obtaining solution to Eq. (20) in a closed form is an open question at this moment and will not be the subject of further consideration in this paper. Instead, the fact that the natural frequency of the free system is known and can be obtained by using Eq. (12) will be employed in order to carry out further analysis by using numerical approach.

Now, with Eq. (12), it is possible to determine natural period of system in Fig. 3 by introducing $\hbar=J_{c} / 2 b$ and $k_{e k v}$ $=m g L$.

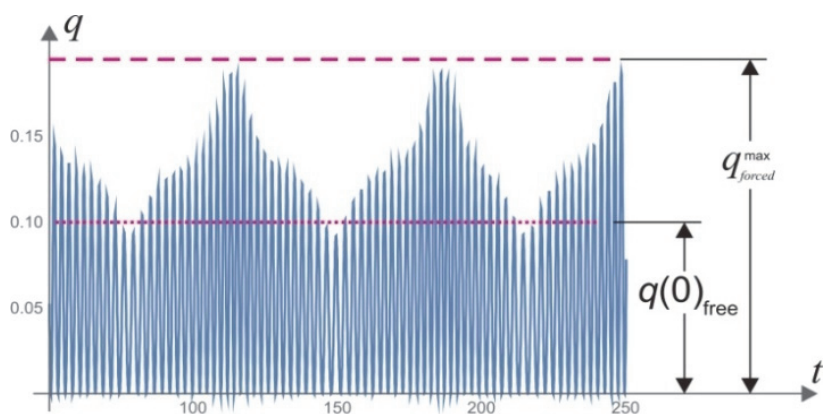

Figure 4 Time history of $q(t)$ derived by numerical integration of Eq. (20) for $m=$ $1 \mathrm{~kg}, L=1 \mathrm{~m}, a=1 \mathrm{~m}, b=0.5 \mathrm{~m}, q(0)=0.1 \mathrm{rad}, \dot{q}(0)=0, X_{0}=0.02 \mathrm{~m}, f_{n}=$ $0.33 \mathrm{~Hz}$ and $\Omega=0.38 \mathrm{~Hz}$

By using this concept, some numerical calculations of Eq. (20) were performed whose results will be shown and discussed subsequently. Time history of generalized coordinate was derived by numerical integration with Wolfram Mathematica [17]. As usual in the field of forced oscillation, it will be shown how the system behaves when subjected to external excitation whose frequency $\Omega$ is close to the natural frequency, as well as when these two frequencies are far from each other.

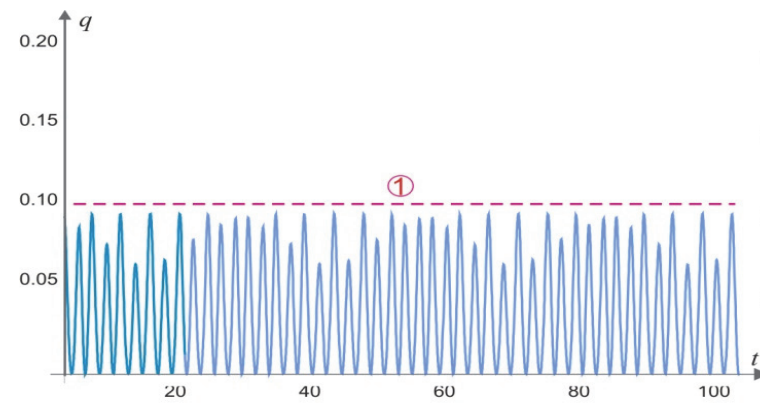

a)

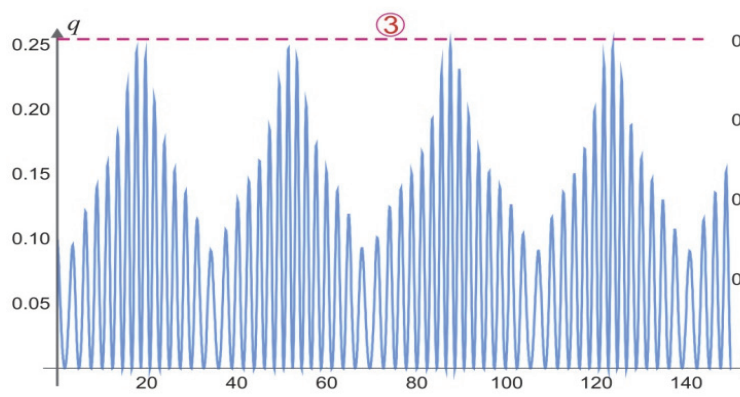

Figure 5 Time history $q(t)$ for: a) $f_{\Omega}=0.171 \mathrm{~Hz} ;$ b) $f_{\Omega}=0.378 \mathrm{~Hz}$; c) $f_{\Omega}=0.415 \mathrm{~Hz}$; d) $f_{\Omega}=0.43 \mathrm{~Hz}$; For all cases: $m=1 \mathrm{~kg}, L=1 \mathrm{~m}, a=1 \mathrm{~m}, b=0.5 \mathrm{~m}, q(0)=0.1 \mathrm{rad}$, $\dot{q}(0)=0.1, X_{0}=0.05 \mathrm{~m}, f_{n}=0.33 \mathrm{~Hz}$

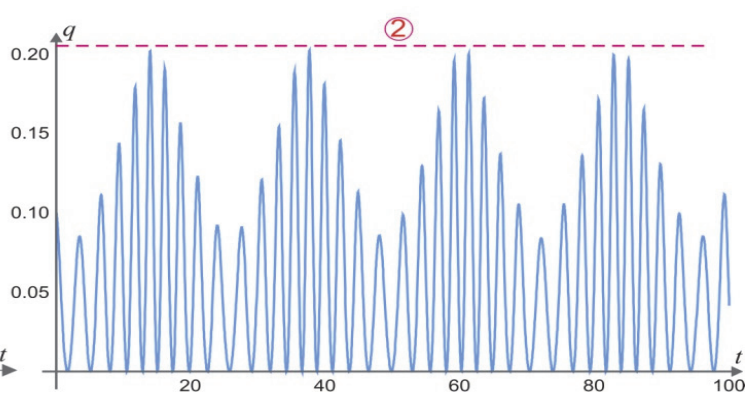

b)

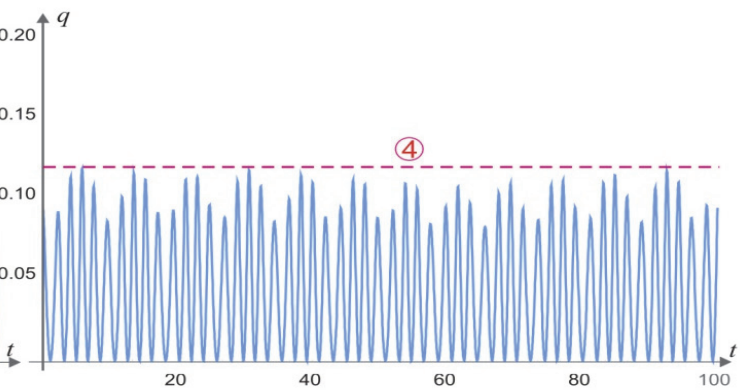

First example of system behaviour at forced oscillations is illustrated by Fig. 4. Worth noticing is characteristic shape of response curve, whose envelope can be described as bulb shaped. This is a novel shape that has not been observed earlier in the field of forced oscillations with harmonic excitation. It should be emphasized that since influence of damping is not considered here (i.e. system is assumed to be conservative), there is no possibility of steady state oscillations. Since free oscillations defined by initial conditions will not vanish due to lack of damping, system responses shown in Fig. 4 and Fig. 5 are influenced by them. In examples considered, zero initial velocity is assumed (which does not impair approach generality), so the maximum amplitude of free oscillations is defined by initial value of generalized coordinate. This value is denoted by $q(0)_{\text {free }}^{\max }$ in Fig. 4 and marked by dotted (red) horizontal line. Maximum values for the motion that results from interaction with the support i.e. from external excitation are denoted by $q(0)_{\text {forced }}^{\max }$ and presented by horizontal dashed lines (Fig. 4 and Fig. 5).

For the purpose of illustration of influence of excitation frequency, numerical analysis has been carried out for four cases, i.e. four different values of excitation frequency (as given by Eq. (13)). Results are shown in Fig. 5 . For adopted values of design parameters (mass of 
platform $m=1 \mathrm{~kg}$, length of platform $L=2 \mathrm{~m}, a=1 \mathrm{~m}$ and $b=0.5 \mathrm{~m}$ ), natural period (Eq. (21)) is $T=3.027 \mathrm{~s}$, i.e. natural frequency is $f_{n}=0.33 \mathrm{~Hz}$. In the case of Fig. 5a, excitation frequency $f_{\Omega}=0.171 \mathrm{~Hz}$ is well below natural frequency. It can be seen that the platform is swinging within the initial angle, so there is no influence of external excitation on natural motion of the platform. The case when $f_{\Omega}$ is close to $f_{n}$ is shown in Fig. $5 \mathrm{~b}$ where the wellknown beating phenomenon can be noticed. Also, due to the influence of periodic external motion, amplitude of coordinate $q$ was significantly increased, as expected for nonlinear forced oscillations.

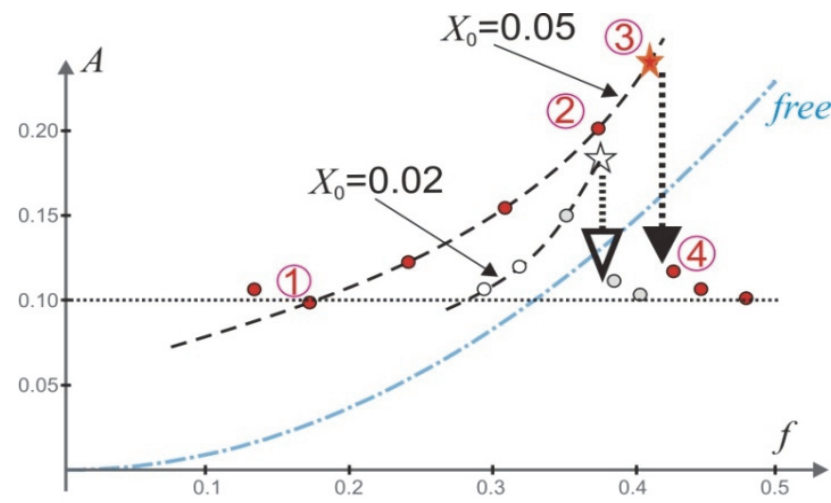

Figure 6 Dependence of amplitude on frequency for cases from Fig. 5. Points 1 2, 3 and 4 correspond to cases a), b), c) and d) from Fig. 5. Dash-dot (blue) line is amplitude-frequency curve for free oscillations.

Fig. 6 shows dependence of peak amplitude on frequency or amplitude-frequency curve (AF), which is important characteristic for non-linear oscillations, for different cases of external conditions. It enables comparison between free system response and system behaviour at forced oscillations by different excitation conditions. Blue dash-dot line represents the backbone curve of the free system. Two black dashed lines represent AF curves of system response for excitation amplitudes of $0.02 \mathrm{~m}$ and $0.05 \mathrm{~m}$ as denoted in figures (cases illustrated by Fig. 4 and Fig. 5). At the line corresponding to $0.05 \mathrm{~m}$ excitation amplitude, solid red dots denoted by 1,2 and 3 are marked. These points correspond to peak amplitudes for the cases 1, 2 and 3 shown in Fig. 5a, Fig. 5b and Fig. $5 \mathrm{c}$ respectively. Point 3 is at the same time marked by the solid red star. At this point system achieves maximum peak of response amplitude for the given excitation amplitude. Characteristic for this point (i.e. system operating regime) is, further, that from here, for the small excitation frequency increase, response amplitude jumps toward significantly smaller values as denoted by the solid red arrow and switches to operating regime at the point 4 (corresponding to case 4 from Fig. 5d. It can be seen from Fig. 6 that the system exhibits qualitatively the same kind of behaviour for excitation amplitude of $0.02 \mathrm{~m}$, but with smaller maximal values of amplitudes. For this second case, maximum possible response amplitude is denoted by the open dots. Phenomenon of downward jump for this case is denoted by the open arrow.

It is worthwhile comparing the system considered herein with the Duffing oscillator [18], since the later is one of the most common examples of nonlinear oscillatory system. Comparison shows that there are both similarities and differences in behaviour between the two systems.
Regarding differences in main system properties, Duffing oscillator has nonlinear restoring force, while restoring force of OHVI is a linear function of generalized coordinate. Similarities include growth of maximum amplitude with excitation frequency increase, as well as characteristic jumps downwards, which can be observed in AF diagram shown in Fig. 6. These jumps indicate that bifurcation of solutions may exist. Further, backbone curve of OHVI grows with frequency (Fig. 6), as well as it is the case for Duffing system. On the other hand, backbone curve of OHVI is of convex shape, as opposed to Duffing system whose backbone curve is concave. It is interesting to note that OHVI system behaves like a hardening Duffing oscillator since its backbone bends upwards with increasing frequency.

\section{CONCLUSION}

In this paper one special class of nonlinear oscillator was introduced and its behaviour considered regarding both free and forced oscillations. Possibility of achieving low i.e. ultra-low natural frequency opens wide field for important engineering applications of such system such as e.g. vibration suppression and many others. Technique that employs system nonlinearity was used to address such issues. Uniqueness of this system stems from the novel form of kinetic energy, not found in previous literature, which reflects the fact that system inertia changes hyperbolically as a consequence of system motion. On the basis of this feature, new notion of Oscillator with Hyperbolically Variable Inertia (OHVI) is introduced. Authors consider that this new way of thinking may be extended to other non-mechanical systems and applied universally, therefore having both theoretical and practical importance for contemporary engineering and science. For this reason considerations began from the general form of systems, not only mechanical, which is another novel feature introduced by this paper.

Another original contribution of this work is the relation between introduced system and harmonic oscillator that was established herein via contraction and deformation of energy surface, also enabling better understanding and insight into system behaviour. One of the main and at the same time most intriguing features of regarded system is that the equilibrium position coincides with the stopping point. Deeper attention was paid to this special feature. Thereby it was noted that the system would be able to carry out fully oscillatory behaviour during time (passing through stopping point and continuing on) only with external energy supplied, since free system motion obeys asymptotic solution. Having in mind that the concrete mechanical system example was presented here as an illustration of regarded class of systems, this discussion may be useful for educational purposes in the scope of theoretical considerations of dynamic systems. Connection established between novel general concept introduced herein and real mechanical system is therefore another very important result of this work.

In the scope of analysis it was demonstrated that with OHVI it is possible to achieve a long period of motion as well as low intensity of velocity with respect to $\mathrm{HO}$ of comparable size. Coincidence of equilibrium and stop position provides an opportunity to establish an analogy 
with overdamped oscillator, although in this case there is no damping. Such behaviour can be viewed as a superattenuation feature. Results obtained for free system give a basis for considerations of forced oscillations. Unlike conservative case, for which exact solution was derived in the form of special Jacobi elliptic function, for nonautonomous systems this approach does not apply because of nonlinear differential equation nonhomogeneity. Therefore, study of several characteristic cases was carried out by providing numerical solutions, based on the possibility to obtain natural frequency of the free system. Mathematical model derived for mechanical system was discussed and further directions for analytic considerations were pointed out. Numerical techniques were employed to illustrate some characteristic forms of nonautonomous system behaviour. Beating phenomenon in the vicinity of the backbone amplitude-frequency curve was demonstrated. By this numerical example it was shown that there are some similarities between regarded system and other nonlinear oscillatory systems that are governed by nonsingular equations. These similarities include phenomena such as bending of the backbone curve, influence of forcing amplitude, or downward jump. Having in mind that forced oscillations with singularity considered here have not been investigated yet, these conclusions presented here can represent sound basis for further theoretical and practical development.

\section{Acknowledgements}

This work was financially supported by the Ministry of Education, Science and Technological Development of the Republic of Serbia (involvement of Z. R.: Project III41007; involvement of B. S.: Projects TR35041 and TR31046).

\section{REFERENCES}

[1] Acton, J. R. \& Squire, P. T. (1985). Solving Equations with Physical Understanding, $1^{\text {st }}$ ed. Adam Hilger, Bristol and Boston

[2] Mickens, R. E. (2007). Harmonic balance and iteration calculations of periodic solutions toy" $+\mathrm{y}^{-1}=0$. J Sound Vib 306, 968-972. https://doi.org/10.1016/j.jsv.2007.06.010

[3] Blair, D. G., Liu, J., Moghaddam, E. F., \& Ju, L. (1994). Performance of an ultra low-frequency folded pendulum. Physics Letters A, 193(3), 223-226. https://doi.org/10.1016/0375-9601(94)90587-8

[4] Starossek, U. (2015). A low-frequency pendulum mechanism. Mech Mach Theory, 83, 81-90. https://doi.org/10.1016/j.mechmachtheory.2014.08.010

[5] Ferguson, E. S. (1962). Kinematics of mechanisms from the Time of Watt. US National Museum Bulletin, 228, 185-230.

[6] Barone, F. \& Giordano, G. (2018). A new typology of DC tiltmeter based on the Watt's linkage architecture. Sensors and Actuators, A: Physical, 281, 264-277. https://doi.org/10.1016/j.sna.2018.08.015

[7] Barone, F. \& Giordano, G. (2018). The UNISA Folded Pendulum: a very versatile class of low frequency high sensitive sensors. Measurement, 118, 339-347. https://doi.org/10.1016/j.measurement.2017.09.001

[8] Kołakowski, P. (2007). Structural health monitoring-a review with the emphasis on low-frequency methods. Engineering Transactions, 55(3), 239-275.
[9] Bennetts, L. G., Peter, M. A., Dylejko, P., \& Skvortsov, A. (2019). Effective properties of acoustic metamaterial chains with low-frequency bandgaps controlled by the geometry of lightweight mass-link attachments. Journal of Sound and Vibration, 456, 1-12. https://doi.org/10.1016/j.jsv.2019.05.022

[10] Hua, C. J., Wang, C. F., Lu, Y. J. (2019). Research on fracture behavior of metal pipe based on high and low frequency composite vibration. Chinese Journal of Engineering Design, 26(2), 223-229.

[11] Fan, K., Zhang, Y., Liu, H., Cai, M., \& Tan, Q. (2019). A nonlinear two-degree-of-freedom electromagnetic energy harvester for ultra-low frequency vibrations and human body motions. Renewable Energy, 138, 292-302. https://doi.org/10.1016/j.renene.2019.01.105

[12] Ibrahim, R. A. (2008). Recent advances in nonlinear passive vibration isolators. Journal of Sound and Vibration, 314, 371-452. https://doi.org/10.1016/j.jsv.2008.01.014

[13] Gatti, G., Brennan, M. J., \& Tang, B. (2019). Some diverse examples of exploiting the beneficial effects of geometric stiffness nonlinearity. Mechanical Systems and Signal Processing, 125, 4-20. https://doi.org/10.1016/j.ymssp.2018.08.024

[14] Rach, R., Baghdasarian, A., \& Adomian, G. (1992). Differential Equations with Singular Coefficients. Appl Math Comput, 47, 179-84. https://doi.org/10.1016/0096-3003(92)90045-3

[15] Jordan, D. W. \& Smith, P. (2007). Nonlinear ordinary differential equations: an introduction for scientists and engineers, $4^{\text {th }} \mathrm{ed}$. Oxford University press, Oxford.

[16] Kovacic, I., Cveticanin, L., Zukovic, M., \& Rakaric, Z. (2016). Jacobi elliptic Function: A review of a nonlinear oscillatory application problems. J Sound Vib, 380, 1-36. https://doi.org/10.1016/j.jsv.2016.05.051.

[17] Wolfram Research Inc. (2018). Mathematica, Version 11.3. Champaign, IL (2018).

[18] Nayfeh, A. H. \& Mook, D. T. (1995). Nonlinear Oscillations. Willey, New York. https://doi.org/10.1002/9783527617586

\section{Contact information:}

Zvonko RAKARIĆ, PhD, Associate Professor University of Novi Sad-Faculty of Technical Sciences, Department of Mechanics, Trg Dositeja Obradovića 6, 21000 Novi Sad-Serbia

E-mail: zvonko@uns.ac.rs

\section{Boris STOJIĆ, PhD, Assistant Professor}

(Corresponding author)

University of Novi Sad-Faculty of Technical Sciences, Department of

Mechanization and Design Engineering,

Trg Dositeja Obradovića 6, 21000 Novi Sad-Serbia

E-mail: bstojic@uns.ac.rs 